\title{
LIST OF PARTICIPANTS
}

C. Arpigny, Institut d'Astrophysique, Cointe-Sclessin, Belgium

C. A. Barth, Laboratory for Atmospheric and Space Physics, Boulder, Colo., U.S.A.

R. C. Bless, Washburn Observatory, Madison, Wis., U.S.A.

J. van Boeckel, European Space Research and Technology Center, Noordwijk, The Netherlands

T. den Boggende, Space Research Laboratory, Utrecht, The Netherlands

A. Boksenberg, University College, London, United Kingdom

G. Boldt, Max Planck-Institut für Physik und Astrophysik, Munich, Germany

R. M. Bonnet, Laboratoire dc Physique stellaire et planétaire, Verrières-le-Buisson, France

J. Borgman, Kapteyn Astronomical Laboratory, Groningen, The Netherlands

B. Brinkman, Space Research Laboratory, Utrecht, The Netherlands

M. Burger, Astronomical Institute, Utrecht, The Netherlands

W. M. Burton, Culham Laboratory, Abingdon, United Kingdom

H. E. Butler, Royal Observatory, Edinburgh, United Kingdom

J. W. Campbell, Royal Observatory, Edinburgh, United Kingdom

A. M. Cantu, Osservatorio Astrofisico di Arcetri, Firenze, Italy

G. R. Carruthers, Naval Research Laboratory, Washington D.C., U.S.A.

T. A. Chubb, Naval Research Laboratory, Washington D.C., U.S.A.

D. D. Clark, European Space Research and Technology Center, Noordwijk, The Netherlands

J. Collet, European Space Research Organisation, Neuilly-sur-Seine, France

P. S. Conti, Lick Observatory, Santa Cruz, Calif., U.S.A.

G. Courtès, Laboratoire d'Astronomie spatiale, Marseille, France

R. J. Davis, Smithsonian Astrophysical Observatory, Cambridge, Mass., U.S.A.

J. Davis, Chatterton Astronomy Department, School of Physics, Sidney, Australia

J. P. Delaboudinière, Laboratoire de Physique stellaire et planétaire, Verrières-leBuisson, France

A. J. Deutsch, Mount Wilson and Palomar Observatories, Pasadena, Calif., U.S.A. N. A. Dimov, Crimean Astrophysical Observatory, Nauchny, Crimea, U.S.S.R.

R. J. van Duinen, Kapteyn Astronomical Laboratory, Groningen, The Netherlands

J. Emming, Space Research Laboratory, Utrecht, The Netherlands

F. Engström, Stockholm Observatory, Saltsjobaden, Sweden

R. Faraggiana, Osservatorio, Trieste, Italy

M. W. Feast, Radcliffe Observatory, Pretoria, South Africa

L. de Feiter, Space Research Laboratory, Utrecht, The Netherlands

K. Fredga, Astronomical Institute, Utrecht, The Netherlands 
M. Fulchignoni, Laboratorio di Astrofisica, Frascati, Italy

O. Gingerich, Smithsonian Astrophysical Observatory, Cambridge, Mass., U.S.A.

L. Gratton, Laboratorio di Astrofisica, Frascati, Italy

J. M. Greenberg, Sterrewacht te Leiden, Leiden, The Netherlands

H. G. Groth, Universitäts-Sternwarte, Munich, Germany

M. Hack, Osservatorio Trieste, Italy

L. Hansen, University Observatory, Copenhagen, Denmark

W. Haupt, Astronomisches Institut, Ruhr-Universität, Bochum, Germany

D. Hayes, Rensselaer Polytechnic Institute, Troy, N.Y., U.S.A.

A. G. Hearn, Observatoire de Nice, France

J. R. Heintze, Astronomical Institute, Utrecht, The Netherlands

J. Hekela, Observatory Ondrejov, Czechoslovakia

K. G. Henize, NASA Manned Space Flight Center, Houston, Tex., U.S.A.

G. H. Herbig, Lick Observatory, Santa Cruz, Calif., U.S.A.

C. Heynekamp, Space Research Laboratory, Utrecht, The Netherlands

A. M. Hieronimus, Centre National d'Études Spatiales, Bretigny-sur-Orge, France

L. Houziaux, Département d'Astrophysique, Faculté des Sciences, Mons, Belgium

H. C. van de Hulst, Sterrewacht te Leiden, Leiden, The Netherlands

C. M. Humphries, Royal Observatory, Edinburgh, United Kingdom

J. Hutchings, Dominion Astrophysical Observatory, Victoria, British Columbia, Canada

C. de Jager, Astronomical Institute, Utrecht, The Netherlands

E. B. Jenkins, Princeton University Observatory, Princeton, N.J., U.S.A.

C. Jordan, Culham Laboratory, Abingdon, United Kingdom

H. E. Jorgensen, University Observatory, Copenhagen, Denmark

B. Kovatschev, Bulgarian Academy of Sciences, Department of Astronomy, Sofia, Bulgaria

L. V. Kuhi, Berkeley Astronomy Department, Berkeley, Calif., U.S.A.

V. G. Kurt, Sternberg Astronomical Institute, Moscow, U.S.S.R.

H. J. Lamers, Astronomical Institute, Utrecht, The Netherlands

M. Lehmann, Observatoire de Genève, Sauverny, Switzerlảnd

P. Lemaire, Laboratoire de Physique stellaire et planétaire, Verrières-le-Buisson, France

M. C. Lortet, Institut d'Astrophysique, Paris, France

D. Malaise, Institut d'Astrophysique, Cointe-Ougrée, Belgium

L. Meredith, Goddard Space Flight Center, Greenbelt, Md., U.S.A.

D. C. Morton, Princeton University Observatory, Princeton, N.J., U.S.A.

E. A. Müller, Observatoire de Genève, Sauverny, Switzerland

C. Navach, Observatoire de Genève, Sauverny, Switzerland

J. B. Oke, California Institute of Technology, Pasadena, Calif., U.S.A.

J. H. Oort, Sterrewacht te Leiden, Leiden, The Netherlands

N. Panagia, Laboratorio di Astrofisica, Frascati, Italy

E. Peytremann, Observatoire de Genève, Sauverny, Switzerland 
S. R. Pottasch, Kapteyn Astronomical Laboratory, Groningen, The Netherlands

F. Praderie, Observatoire de Meudon, Meudon, France

V. K. Prokofiev, Crimean Astrophysical Observatory, Nauchny, Crimea, U.S.S.R.

J. Provost, Observatoire de Nice, France

N. K. Reay, Culham Laboratory, Abingdon, United Kingdom

J. B. Rogerson Jr, Princeton University Observatory, Princeton, N.J., U.S.A.

N. G. Roman, NASA Headquarters, Washington D.C., U.S.A.

R. J. Rutten, Astronomical Institute, Utrecht, The Netherlands

D. Samain, Laboratoire de Physique stellaire et planétaire, Verrières-le-Buisson, France

M. Schneider, Observatoire de Nice, France

A. Severny, Crimean Astrophysical Observatory, p/o Nauchny, Crimea, U.S.S.R.

A. M. Smith, Goddard Space Flight Center, Greenbelt, Md., U.S.A.

A. Snijders, Astronomical Institute, Utrecht, The Netherlands

L. Spitzer Jr, Princeton University Observatory, Princeton, N.J., U.S.A.

P. Solomon, Columbia University, Department of Astronomy, New York, N.Y., U.S.A.

T. P. Stecher, Goddard Space Flight Center, Greenbelt, Md., U.S.A.

G. C. Sudbury, Royal Observatory, Edinburgh, United Kingdom

R. A. Sunyaev, Institute of Applied Mathematics, Moscow, U.S.S.R.

J. P. Swings, Institut d'Astrophysique, Cointe-Ougrée, Belgium

G. L. Tagliaferri, Observatorio Astrofisico di Arcetri, Firenze, Italy

V. M. Tiyt, Institute of Physics and Astronomy, Tartu, Estonia, U.S.S.R.

A. B. Underhill, Astronomical Institute, Utrecht, The Netherlands

J. C. Vial, Laboratoire de Physique stellaire et planétaire, Verrières-le-Buisson, France

R. Viotti, Laboratorio di Astrofisica, Frascati, Italy

H. Visser, Astronomical Institute, Utrecht, The Netherlands

M. Viton, Laboratoire d'Astronomie spatiale, Marseille, France

G. Walker, University of British Columbia, Vancouver, B.C., Canada

R. M. West, University Observatory, Copenhagen, Denmark

N. C. Wickramasinghe, Institute of Theoretical Astronomy, University of Cambridge, Cambridge, United Kingdom

R. Wilson, Culham Laboratory, Abingdon, Berkshire, United Kingdom

H. van Woerden, Kapteyn Astronomical Laboratory, Groningen, The Netherlands 